\title{
Subjective versus objective assessments of sleep among middle aged male patients after coronary artery bypass grafting: A correlational study
}

\author{
Hady Atef $^{*}$, Zeinab Helmy ${ }^{1}$, Abeer Ahmed Farghaly¹, Sameh Elameen²
}

1 Faculty of physical therapy, Cairo University. Egypt

2 National Heart Institute of Egypt, Egypt

\begin{abstract}
Background: Sleep quality is a complex construct to evaluate empirically, and yet the Pittsburg Sleep Quality Index (PSQI) is commonly used in studies as their only measure of sleep quality. Furthermore, the PSQI may not be the best sleep quality measure for older adults post coronary artery bypass graft (CABG), due to its reliance on the cognitive capacity to reflect on the past month. Purpose: The present study was conducted to compare the PSQl's predictive validity as a subjective measure of sleep quality with an objective sleep quality (as measured by actigraphy). Methods: 80 male participants aged 45-65years-who underwent coronary artery bypass graft CABG since 6 weeks - were randomized to two groups: aerobic group (AG), and combined aerobic and resistance group (ARG). Training lasted ten consecutive weeks with 30 uninterrupted sessions. The actigraph together with Pittsburg sleep quality index (PSQI) were used in sleep quality assessment. The actigraph (Actiwatch Minimitter Company, Incorporated (INC) - Sunriver, OR, USA) was placed on the non-dominant wrist and activities were monitored continuously while being recorded at one minute intervals. The participants kept the device for a period of 96 hours, filled PSQI, and did 6MWT before the first and last training sessions. Pearson's correlation was used to assess the relationship between the data obtained from these 2 assessment methods. Results: Our results suggest perceived sleep quality is quite different from objective reality, at least for this group. Previous studies have shown the PSQl to be a valuable tool for assessing subjective sleep quality; however, our findings indicate for middle aged adults who did CABG the PSQI should not be used as a substitute for actigraphy, or vice versa. Conclusion: The best practice is to include both subjective and objective measures when examining sleep quality in older adults (i.e., the PSQI,and actigraphy).

Keywords: Sleep quality, exercise, CABG, actigraphy, PSQI.
\end{abstract}

\section{Introduction}

Changes in sleep patterns after CABG are related to several factors: individual (e.g., age, gender), physiological (e.g., primary sleep disorders, cardiac function, pain, dyspnea, fatigue, nocturia), psychological (e.g., emotional) and environmental (e.g., hospital environment, patient care activities)(P. L. Yang, Huang, Tsai, \& Lou, 2015)

Correspondence: hady612@hotmail.com, +201119699110

Madinaty 113/30/53, New Cairo, Cairo, Egypt

Received: 13 April 2019 Accepted: 05 July 2019

Sleep and Hypnosis

Journal homepage:

http://www.sleepandhypnosis.org

ISSN:1302-1192 (Print) 2458-9101 (Online)
Sufficient sleep is essential for optimum healthjust a few of the numerous processes occurring during sleep include memory consolidation, clearance of brain metabolites, and restoration of nervous, immune, skeletal, and muscular systems. (Kline, 2014) Poor sleep quality might increase the cardiovascular disease (CVD) risk. Interventions to promote the cardiovascular health may need to include sleep promotion strategies. (Chair et al., 2017)

Sleep and exercise affect each other through complex, bidirectional interactions that involve different physiological and psychological pathways. (Peppard et al., 2013) and because of the ongoing obesity epidemic, previous estimates of sleep-disordered breathing prevalence require updating. We estimated the prevalence of sleep-disordered breathing in the United States for the periods of 1988-1994 and 2007-2010 using data from 
the Wisconsin Sleep Cohort Study, an ongoing community-based study that was established in 1988 with participants randomly selected from an employed population of Wisconsin adults. A total of 1,520 participants who were 30-70 years of age had baseline polysomnography studies to assess the presence of sleep-disordered breathing. Participants were invited for repeat studies at 4-year intervals. The prevalence of sleep-disordered breathing was modeled as a function of age, sex, and body mass index, and estimates were extrapolated to US body mass index distributions estimated using data from the National Health and Nutrition Examination Survey. The current prevalence estimates of moderate to severe sleep-disordered breathing (apnea-hypopnea index, measured as events/hour, $\geq 15$ Although physical activity is usually considered a useful tool in sleep management, this link may be subject to multiple factors such as sex, age, fitness level, sleep quality and the characteristics of the exercise used (i.e. intensity, duration, and type).(de Vries, van Hooff, Geurts, \& Kompier, 2016) It is therefore vital to improve knowledge in fundamental physiology in order to understand the benefits of exercise on the quantity and quality of sleep in healthy subjects and patients. (Mendelson, Borowik, Michallet, Perrin, \& Monneret, 2015)

A consistent epidemiologic and experimental evidence can strongly support the significant association between exercise and sleep, and to which degree exercise training (EXT) can significantly improve sleep (Chennaoui, Arnal, Sauvet, \& Léger, 2015)

A two-parallel arm study on 97 subjects to investigate to what extent a low-intensity exercise intervention is effective in improving sleep quality, self-efficacy, and physical fitness. Results underlined the value of low-intensity exercise for subjects with high levels of study-related problems. (de Vries et al., 2016)

Meta-analysis conducted by Yang et al. (P. Yang, Ho, Chen, \& Chien, 2012) investigated how regular exercise impacts sleep structure in middle-aged and older adults with sleep complaints. Results indicated moderate effects in the favourable direction for regular exercise on sleep structure, and determined the acute and chronic effects of resistance exercise on sleep quantity and quality. Chronic resistance exercise improves all aspects of sleep, with the greatest benefit for sleep quality. (Kovacevic, Mavros, Heisz, \& Fiatarone Singh, 2018).
Literature has many theories regarding the mechanism of exercise on sleep structure improvement, explanations including: Thermogenic effect, anxiety reduction, increase in serotonin and subsequent antidepressant effects, immunologic alternations, and improvement in quality of life, (Passos, Dalva, Rollemberg, Gonc, \& Tufik, 2012)(Akbari Kamrani, Shams, Shamsipour Dehkordi, \& Mohajeri, 2014)(Montgomery, Ja, Montgomery, \& Ja, 2009)(Rn et al., 2019), and many other explanations for improvement of functional capacity -which is closely correlated with sleep structure -(Chien \& Chen, 2015) with exercise including: cardiac remodeling, cardiac growth and vascular remodeling due to cellular and molecular adaptations in response to exercise. (Baraúna, Oliveira, Phillips, Negrão, \& Fernandes, 2015)

One widely used self-reported measure of sleep quality, the Pittsburgh Sleep Quality Index (PSQI), has been established as a valid scale with acceptable psychometric properties when used among different population. (Zhong, Gelaye, Sánchez, \& Williams, 2015) PSQI had internal consistency, internal homogeneity, and diagnostic characteristics. This supports the applicability and certain aspects of the validity of the PSQI in the population. (Manzar et al., 2015)

Actigraph has been widely used to measure sleep (Adkins et al., 2012). The operational premise is that periods of sleep are accompanied by minimal movement, and periods of wakefulness are accompanied by relatively more movement, and that such movement can be detected by an accelerometry based activity monitor. (Nichols, 2015) Actigraph worn on the hip does not provide valid or accurate measures of sleep, mainly due to poor wake detection. (Bonardi et al., 2016) Relative to the hip, a wrist worn actigraph provided more valid measures of sleep, but with only moderate capability to detect periods of wake during the sleep period. (Walsh et al., 2015)

Actigraph provided similar accuracies for sleep/ wake determination in subjects as lab based measurments (Stingeni, Raponi, Hansel, Agostinelli, \& Lisi, 2011) Recent findings indicate for older adults the PSQI should not be used as a substitute for actigraphy, or vice versa. Hence, best practice is to include both subjective and objective measures when examining sleep quality in older adults (i.e., the PSQI, and actig- 
raphy) (Landry, Best, \& Liu-Ambrose, 2015)

The objective of this correlational study was to determine whether data got from actigraph can be clinically correlated with those got from PSQI, and consequently can one method of them be used as a substitute for the other for the purpose of sleep assessment, or no tool of them can be used separately to give a full picture about sleep assessment.

\section{METHODS}

The investigation conforms with the principles outlined in the Declaration of Helsinki" (Br Med J 1964; ii: 177). Approval of Research Ethical committee of faculty of physical therapy, Cairo University was taken for this study on 3/12/2017 under number: P.T.REC/012/001805 and a written informed consent for patient information and images to be published was provided by the patients.

\section{I-Patients}

Eighty male patients $(\mathrm{n}=80)$ who have underwent CABG surgery since 6-8 weeks - to make sure that the insomnia is not hospitalization related -were included in this two-parallel arm study. They aged from 45-65. They were recruited during a time period of 6 months from the cardiac surgery department at the National heart institute in Cairo, Egypt and randomization was performed using the sealed envelope approach, to two groups with allocation ratio of 1:1. The two groups are aerobic group (AG) and combined aerobic and resistance group (ARG).

\section{Inclusion criteria}

Patients who had underwent CABG surgery since 6-8 weeks, their ages ranges from 45 and 65years old,medically stable, did CABG-only treatment (not combined with valve replacement surgery), current complaint of poor sleep quality (SQ) (score $>2$ of the SQ scale) and with body mass index $\leq 35 \mathrm{~kg} / \mathrm{m}^{2}$ (i.e. no obstructive sleep apnea) were only included in the study.

\section{Exclusion criteria}

Any patient was known to have any unstable medical condition; history of chronic insomnia for at least one year before surgery, indicated for receiving treatments for depression and/or anxiety or sleep medications, or with any known musculoskeletal or neurological conditions that might interfere with the execution or the assessment of the exercise, was excluded from the study.

\section{II- Instrumentation}

\section{A-Evaluation methods}

The Pittsburgh Sleep Quality Index (PSQI) is a self-report questionnaire that assesses sleep quality over a 1-month time interval. (Mollayeva et al., 2016) It used in this study to assess Sleep quality subjectively.

\section{Actigraphy}

Actigraphy is a valid tool to assist in determining sleep patterns in normal, healthy adult populations, normal infants and children, and patients suspected of certain sleep disorders. (Ancoli-israel et al., 2003) It is used in this study to assess sleep Latency, Total sleep duration, Deep sleep duration, light sleep duration, Sleep Fragmentation index, cutpoints on actigraphy, and Sleep efficiency objectively. (Figure 1)
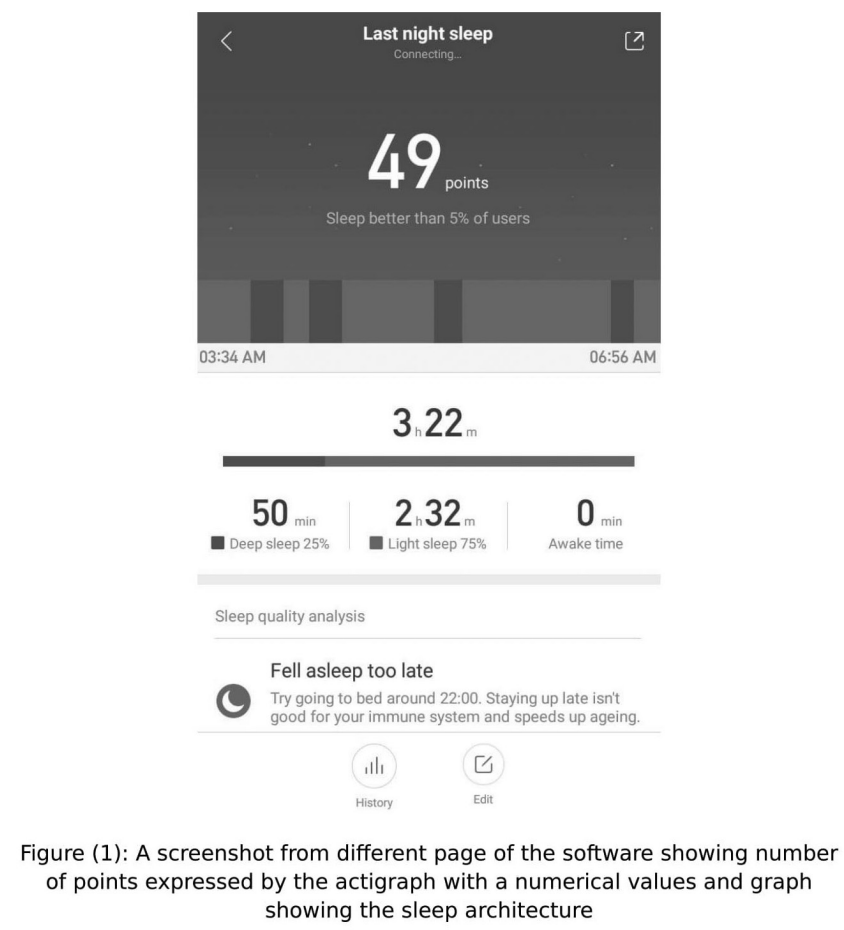

\section{Six minute walk test (6MWT)}

The 6 Minute Walk Test is a sub-maximal exercise test used to assess aerobic capacity and endurance. The distance covered over a time of 6 minutes is used as the outcome by which to compare changes in functional capacity. (Bohannon \& Crouch, 2017) 


\section{Rate of perceived exertion (RPE)}

The RPE scale is used to measure the intensity of exercise. The modified Borg RPE scale is a numerical scale that ranges from 6 to 20, where 6 means "no exertion at all" and 20 means "maximal exertion." When a measurement is taken, a number is chosen from the following scale by an individual that best describes their level of exertion during physical activity. (Mane \& Memushaj, 2018)

RPE is a subjective method showing the level of exertion done by the participant. It was used as an associative method with Training heart rate plotted on the treadmill during the training sessions, and the resistance trainings, to determine the proper exercise intensity, because training heart rate may be a misleading parameter in some cardiac cases who are under beta-blockers, and this was the majority of our cases. Beta blockers may interfere with the normal response of the cardiac muscle to exercise, accordingly, depending solely on heart rate in this situation would be misleading and risky as well, so we use RPE as an alternative method, to make sure that the patient is not exerting much effort beyond the effort needed in the study. (Chen, Fox, Ku, \& Chang, 2016)

Therefore, we depend more -regarding heart rate- on resting heart rate plotted at the beginning of each session as a determinant of the cardiovascular fitness (i.e. resting heart rate decrease in case of increased fitness and vice versa) (Chang, Lin, Ho, \& Huang, 2019)

\section{III-Procedures}

Awere assessed before the first and last sessions via 6MWT to assess their functional capacity, $1 \mathrm{RM}$ to assess the resistance exercise intensity used, PSQI questionnaire and wrist actigraph (Actiwatch Minimitter Company, INC - Sunriver, OR, USA) to assess their sleep archi- tecture. Actigraph was placed on the non-dominant wrist and activities were monitored continuously and recorded at one minute intervals during wakefulness and sleep. The times of wakefulness and sleep were determined individually by means of a diary kept by the volunteers, together with the times calculated by the actigraph monitor. The participants kept the device for a period of 96 hours(Bonardi et al., 2016) before the first and the last training session. The device was removed only at bath time and replaced to the wrist immediately after. The information was collected by the device and recorded by a validated algorithm (Minimitter Company - USA $($ ) and transferred to closed technology software installed in a computer. After analysis of the exams, the data were plotted on a spreadsheet for statistical treatment.

\section{Statistical Analysis}

The data were collected from patients and classified into paired and independent "t" test values. Data will be statistically described in terms of mean \pm standard deviation $( \pm$ SD).

\section{RESULTS}

\section{I- Patients clinical and demographic characteristics}

Eighty male patients $(\mathrm{n}=80)$ with $\mathrm{CABG}$ were included in the study, 40 received aerobic exercise training program (AG) and another 40 received combined aerobic and resistance exercise training program (ARG). Their mean \pm SD values for age, weight, height (in meter), BMI, resting heart rate (in beat/min), and duration post-CABG (6-8) weeks were calculated and there was non-significant difference between both groups (p>0.05). (Table 1)

Table (1): Patients demographic characteristics (Mean \pm Standard Deviation)

\begin{tabular}{|c|c|c|c|c|c|}
\hline Variable & $\begin{array}{l}\text { The AG group } \\
\qquad(n=40)\end{array}$ & $\begin{array}{l}\text { The ARG group } \\
\qquad(n=40)\end{array}$ & $T$ & $\mathbf{P}$ & \\
\hline Age (year) & $54.17 \pm 6.52$ & $53.65 \pm 4.79$ & 0.31 & 0.76 & NS \\
\hline Weight (kg) & $90.7 \pm 6.36$ & $90.04 \pm 5.38$ & 0.38 & 0.71 & NS \\
\hline Height (meter) & $1.69 \pm 0.03$ & $1.67 \pm 0.02$ & 1.81 & 0.08 & NS \\
\hline $\begin{array}{l}\text { Body mass index } \\
\qquad\left(\mathrm{kg} / \mathrm{m}^{2}\right)\end{array}$ & $31.92 \pm 2.58$ & $32.22 \pm 2.3$ & $-0.41-$ & 0.68 & NS \\
\hline $\begin{array}{l}\text { Duration of CABG } \\
\text { (weeks) }\end{array}$ & $6.96 \pm 0.83$ & $7 \pm 0.85$ & $-0.18-$ & 0.86 & NS \\
\hline
\end{tabular}


Table (2): Within and between-groups comparison of evaluated variables.

\begin{tabular}{|c|c|c|c|c|}
\hline \multirow{2}{*}{ Character } & \multirow{2}{*}{$\begin{array}{c}\text { Aerobic Exercise } \\
\text { Training group } \\
\qquad(\mathrm{n}=40)\end{array}$} & \multirow{2}{*}{$\begin{array}{c}\text { Combined Exercise } \\
\text { training group } \\
(n=40)\end{array}$} & \multicolumn{2}{|c|}{ Between Groups Comparison } \\
\hline & & & F-Value & P-Value \\
\hline Resting HR Pre & $100.22 \pm 7.31$ & $106.3 \pm 14.16$ & 1.83 & 0.7 \\
\hline Resting HR Post & $76.09 \pm 7.25$ & $86.3 \pm 10.14$ & 3.93 & 0.0003 \\
\hline$T$ and $P$ values & $15.78,000$ & 12.99 .00 & & \\
\hline Sleep Latency min Pre & $111.52 \pm 83.98$ & $90.87 \pm 36.42$ & 1.08 & 0.29 \\
\hline Sleep latency min Post & $13.7 \pm 10.03$ & $37.17 \pm 16.43$ & 5.85 & 0.00 \\
\hline$T$ and $P$ values & $5.82,0.00$ & $8.98,00$ & & \\
\hline Total_sleep_duration_hours_Pre & $4.28 \pm 0.96$ & $3.98 \pm 1.06$ & 1.02 & 0.31 \\
\hline Total_sleep_duration_hours_Post & $7.78 \pm 1.41$ & $5.17 \pm 1.4$ & 6.28 & 000 \\
\hline$T$ and $P$ values & $-15.59-, 00$ & $-3.45-, 0.002$ & & \\
\hline Deep sleep duration hours Pre & $0.65 \pm 0.24$ & $1.3 \pm 0.77$ & 0.1 & 0.92 \\
\hline Deep sleep duration Post & $6.52 \pm 1.59$ & $3.7 \pm 1.33$ & 6.54 & 000 \\
\hline$T$ and $P$ values & $-15.11-, 00$ & $-6.98-, \quad 0.00$ & & \\
\hline Light sleep duration hours Pre & $3.52 \pm 1.11$ & $3.39 \pm 0.97$ & 0.43 & 0.67 \\
\hline Light sleep duration Post & $1.26 \pm 0.45$ & $1.48 \pm 0.59$ & 1.4 & 0.17 \\
\hline$T$ and $P$ values & $11.12,00$ & $7.88,00$ & & \\
\hline Sleep Fragmentation index Pre & $32.65 \pm 14.7$ & $33.45 \pm 21.88$ & 0.15 & 0.89 \\
\hline Sleep Fragmentation index Post & $8.41 \pm 2.03$ & $14.98 \pm 4.37$ & 6.54 & 000 \\
\hline$T$ and $P$ values & $7.19,00$ & $3.94,0.001$ & & \\
\hline Cutpoints on actigraph Pre & $56 \pm 11.45$ & $56.96 \pm 7.65$ & 0.33 & 0.74 \\
\hline Cutpoints on actigraph Post & $134.7 \pm 15.89$ & $97.74 \pm 13.94$ & 8.38 & 0.000 \\
\hline$T$ and $P$ values & $-19.11,00$ & $-14.12,00$ & & \\
\hline Sleep efficiency Pre & $35.17 \pm 19.03$ & $35.26 \pm 26.73$ & 0.01 & 0.99 \\
\hline Sleep efficiency Post & $86.65 \pm 3.23$ & $76.22 \pm 6.94$ & 6.54 & 000 \\
\hline$T$ and $P$ values & $-13.95,00$ & $-7.45,00$ & & \\
\hline Functional capacity meter Pre & $130.13 \pm 44.2$ & $128.7 \pm 89.19$ & 0.07 & 0.95 \\
\hline Functional capacity Post & $450.65 \pm 21$ & $429 \pm 35.733865$ & 2.51 & 0.02 \\
\hline$T$ and $P$ values & $-37.27,00$ & $-19.47,00$ & & \\
\hline
\end{tabular}

Level of significance at $P<0.05$

Figure (2): Percent distribution of sleep quality in both groups (pre and post-study)

AG: aerobic exercise training group, ARG: the combined exercise training group.

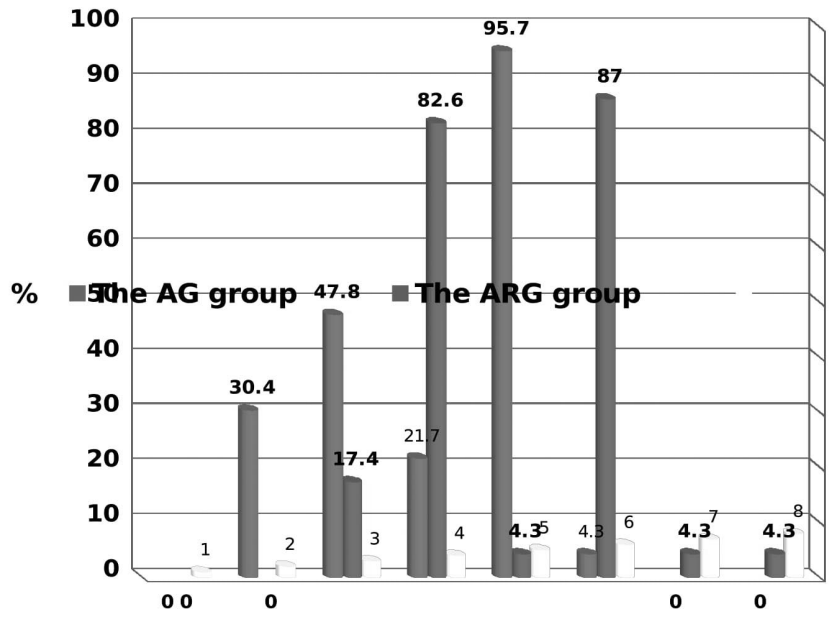




\section{II- Exercise data}

Sleep and functional capacity measurements: In spite that there was significant decrease in the mean value of Sleep Latency, fragmentation index, light sleep duration and sleep quality scores (3=very bad, $2=$ bad, $1=$ good and $\mathrm{O}=$ very good $)(\mathrm{P}<0.01)$ and significant increase in total sleep duration, deep sleep duration, cutpoints gained on the actigraphy, sleep efficiency and functional capacity in both the aerobic group (AG) and combined aerobic and resisted group (ARG) $(\mathrm{P}<0.01)$, that difference was more significant in the aerobic exercise training group $(\mathrm{AG})(\mathrm{P}<\mathrm{O} .01)$ (Table 2), (Figure 2), (Figure 3)

\section{III- Correlation between sleep measurements obtained from PSQI and actigraph in AG group}

Correlation analysis between Sleep variables including (sleep Latency, Total sleep duration, Deep sleep duration, light sleep duration, Sleep Fragmentation index, cutpoints on actigraphy, Sleep efficiency, Sleep quality), revealed that:
There was no significant correlation between sleep quality (measured by PSQI) and any of the sleep variables measured by actigraph. (Table 3)

\section{VI-Correlation analysis between evaluated variables' values in the the combined exercise training group (ARG):}

Correlation analysis between Sleep variables including (sleep Latency, Total sleep duration, Deep sleep duration, light sleep duration, Sleep Fragmentation index, cutpoints on actigraphy, Sleep efficiency, Sleep quality), revealed that:

There was no significant correlation between sleep quality (measured by PSQI) and any of the sleep variables measured by actigraph except light sleep duration. (Table 4)

\section{DISCUSSION}

Coronary artery bypass graft (CABG) surgery is known to be the most frequently performed heart sur-

Table (3) Pearson Correlation analysis between evaluated variables in the aerobic exercise training group (AG).

(Pearson correlation coefficient "r", significance "p value")

\begin{tabular}{|c|c|c|c|c|c|c|c|c|c|c|c|}
\hline \multicolumn{2}{|l|}{ Variable } & RHR & FC & SL & TSD & DSD & LSD & SFI & NPA & SE & SQ \\
\hline \multirow{2}{*}{ FC: Functional Capacity } & $(r)$ & 0.48 & & & & & & & & & \\
\hline & P-value & 0.2 & & & & & & & & & \\
\hline \multirow{2}{*}{ SL: Sleep Latency } & $(r)$ & 0.37 & 0.35 & & & & & & & & \\
\hline & P-value & 0.09 & 0.1 & & & & & & & & \\
\hline \multirow{2}{*}{ TSD: Total sleep duration } & $(r)$ & 0.2 & 0.11 & 0.81 & & & & & & & \\
\hline & P-value & 0.37 & 0.64 & 0.00 & & & & & & & \\
\hline \multirow{2}{*}{ DSD: Deep sleep duration } & $(r)$ & 0.06 & 0.1 & 0.9 & 0.96 & & & & & & \\
\hline & P-value & 0.78 & 0.67 & 0.00 & 0.00 & & & & & & \\
\hline \multirow{2}{*}{ LSD: light sleep duration } & $(r)$ & 0.4 & 0.01 & 0.63 & 0.27 & 0.52 & & & & & \\
\hline & P-value & 0.06 & 0.97 & 0.001 & 0.22 & 0.01 & & & & & \\
\hline \multirow{2}{*}{ SFI: Sleep Fragmentation index } & $(r)$ & 0.33 & 0.18 & 0.42 & 0.77 & 0.64 & 0.16 & & & & \\
\hline & P-value & 0.13 & 0.42 & 0.4 & 0.00 & 0.001 & 0.47 & & & & \\
\hline \multirow{2}{*}{ CPA: Cutpoints on actigraphy } & $(r)$ & 0.003 & 0.25 & 0.92 & 0.96 & 0.98 & 0.45 & 0.59 & & & \\
\hline & P-value & 0.99 & 0.26 & 0.00 & 0.00 & 0.00 & 0.03 & 0.003 & & & \\
\hline \multirow{2}{*}{ SE: Sleep efficiency } & $(r)$ & 0.33 & 0.18 & 0.42 & 0.77 & 0.64 & 0.16 & 1.00 & 0.59 & & \\
\hline & P-value & 0.13 & 0.42 & 0.4 & 0.00 & 0.001 & 0.47 & 0.00 & 0.003 & & \\
\hline \multirow{2}{*}{ SQ: Sleep quality } & $(r)$ & 0.27 & 0.5 & 0.14 & 0.03 & 0.07 & 0.36 & 0.11 & 0.05 & 0.11 & \\
\hline & P-value & 0.22 & 0.84 & 0.53 & 0.88 & 0.75 & 0.9 & 0.61 & 0.84 & 0.61 & \\
\hline
\end{tabular}

Level of significance at $\mathrm{P}<0.05$, (r): Correlation coefficient (r), RHR: Resting Heart Rate, FC: Functional Capacity, SL: Sleep Latency, TSD: Total sleep duration, DSD: Deep sleep duration, LSD: light sleep duration, SFI: Sleep Fragmentation index, CPA: Cutpoints on actigraphy, SE: Sleep efficiency, SQ: Sleep quality. 
Table (4) Pearson Correlation analysis between evaluated variables in the Combined Exercise training group (ARG). (Pearson correlation coefficient " $r$ ", significance "p value")

\begin{tabular}{|c|c|c|c|c|c|c|c|c|c|c|c|}
\hline \multicolumn{2}{|l|}{ Variable } & RHR & FC & SL & TSD & DSD & LSD & SFI & NPA & SE & SQ \\
\hline \multirow{2}{*}{ FC:Functional Capacity } & $(r)$ & 0.78 & & & & & & & & & \\
\hline & P-value & 0.00 & & & & & & & & & \\
\hline \multirow{2}{*}{ SL: Sleep Latency } & $(r)$ & 0.83 & 0.72 & & & & & & & & \\
\hline & P-value & 0.00 & 0.00 & & & & & & & & \\
\hline \multirow{2}{*}{ TSD: Total sleep duration } & $(r)$ & 0.7 & 0.48 & 0.31 & & & & & & & \\
\hline & P-value & 0.00 & 0.02 & 0.15 & & & & & & & \\
\hline \multirow{2}{*}{ DSD: Deep sleep duration } & $(r)$ & 0.52 & 0.24 & 0.12 & 0.91 & & & & & & \\
\hline & P-value & 0.01 & 0.28 & 0.6 & 0.00 & & & & & & \\
\hline \multirow{2}{*}{ LSD: light sleep duration } & $(\mathrm{r})$ & 0.5 & 0.6 & 0.47 & 0.33 & 0.1 & & & & & \\
\hline & P-value & 0.02 & 0.003 & 0.02 & 0.12 & 0.67 & & & & & \\
\hline \multirow{2}{*}{ SFI: Sleep Fragmentation index } & $(r)$ & 0.3 & 0.13 & 0.03 & 0.74 & 0.9 & 0.26 & & & & \\
\hline & P-value & 0.16 & 0.57 & 0.88 & 0.00 & 0.00 & 0.23 & & & & \\
\hline \multirow{2}{*}{ CPA: Cutpoints on actigraphy } & $(r)$ & 0.49 & 0.29 & 0.03 & 0.9 & 0.98 & 0.07 & 0.82 & & & \\
\hline & P-value & 0.02 & 0.18 & 0.88 & 0.00 & 0.00 & 0.76 & 0.00 & & & \\
\hline \multirow{2}{*}{ SE: Sleep efficiency } & $(r)$ & 0.3 & 0.13 & 0.03 & 0.74 & 0.9 & 0.26 & 1.00 & 0.82 & & \\
\hline & P-value & 0.16 & 0.57 & 0.87 & 0.00 & 0.00 & 0.23 & 0.00 & 0.00 & & \\
\hline \multirow[t]{2}{*}{ SQ: Sleep quality } & $(r)$ & 0.24 & 0.1 & 0.05 & 0.09 & 0.36 & 0.6 & 0.27 & 0.38 & 0.27 & \\
\hline & P-value & 0.27 & 0.64 & 0.82 & 0.7 & 0.09 & 0.002 & 0.21 & 0.7 & 0.21 & \\
\hline
\end{tabular}

Level of significance at P<0.05, (r): Correlation coefficient (r), RHR: Resting Heart Rate, FC: Functional Capacity, SL: Sleep Latency, TSD: Total sleep duration, DSD: Deep sleep duration, LSD: light sleep duration, SFI: Sleep Fragmentation index, CPA: Cutpoints on actigraphy, SE: Sleep efficiency, SQ: Sleep quality.

gery on adults. (Joseph, Whitcomb, \& Taylor, 2015) Disturbed sleep, and persistent stress in the postoperative period related to potential death of the loved one and the burden of chronic illness are associated with the development of psychiatric and physical illness (Barger et al., 2017)

Sleep Deprivation is capable of inducing different biological effects, such as neural autonomic control changes, increased oxidative stress, altered inflammatory and coagulatory responses and accelerated atherosclerosis, and all these mechanisms links SD and cardiovascular and metabolic disorders. Epidemiological studies have shown that short sleep duration is associated with increased incidence of cardiovascular diseases, such as coronary artery disease, hypertension, arrhythmias, diabetes and obesity, after adjustment for socioeconomic and demographic risk factors and comorbidities. (Tobaldini et al., 2017)such as insomnia, obstructive sleep apnea (OSA

This study aimed to find an adjusted protocol for assessment of sleep deprivation that may happen after CABG surgery.
The sleep parameters were measured via PSQI questionnaire and actigraph at the beginning and at the end of the study for both aerobic and combined aerobic and resistance groups, and functional capacity was measured by 6MWT at equivalent time-points (before the start and after 10 weeks).

This study demonstrated using one method of assessment of sleep is not enough to give realistic full picture about the sleep, and both a subjective measure like PSQI has to be used in conjunction with objective tool like actigraph for proper sleep assessment in middle aged male patients post CABG surgery.

In our study, we showed that actigraph is a sensitive measurement providing unique information not captured by self-report, and may be an informative adjunct to self-report sleep assessments. This result comes in agreement with Avery et al who assessed sleep in Thirty-six ultra-high risk (UHR) adolescents and 31 healthy control youth participated in a baseline evaluation including 5 nights of actigraphy, subjective measurement of sleep health (Pittsburgh Sleep Quality Index; PSQI), and clinical interviews. Clinical 
measures were repeated with UHR youth $(\mathrm{N}=23)$ at a 12-month follow-up. The results suggest a potential role for actigraphy in sleep assessment beside subjective measures.(Lunsford-Avery, LeBourgeois, Gupta, \& Mittal, 2015)

Slightam et al. (Slightam et al., 2017) Compared self-report and actigraphic evaluations of sleep between veterans with post-traumatic stress disorder and controls, where participants reported their sleep retrospectively for the month before the recording night and on the recording night. On the recording night, they wore an Actiwatch-64 and were instructed to press the marker button upon getting into bed, each time they awoke, and at their final awakening. Results showed that diary-reported number of awakenings was fewer than actigraphically captured awakenings. These results suggest a memory bias towards remembering worse sleep on the nights before the recording night and recommend the use of both methods together.

Some studies have other opinions, and saw that using PSQI is more valid and reliable than actigraph for assessment of sleep, like the study of Fontes et al (Fontes et al., 2017) forward uncertainty propagation is frequently used, whereas backward uncertainty propagation is rarely used. In forward uncertainty analysis, one moves from a given (or assumedwhich assessed the factor structure, internal consistency, test-retest reliability, and construct validity of the European Portuguese version of the Pittsburgh Sleep Quality Index (PSQI) in breast cancer patients against actigraph measurments and proved that the unidimensional construct of the European Portuguese version of the PSQI showed adequate reliability and validity against actigraphic measurements among breast cancer patients.

Another study conducted by Zhu et al stated (Zhu, Bronas, \& Fritschi, 2018) that the agreement between

\section{References}

Adkins, K. W., Goldman, S. E., Fawkes, D., Surdyka, K., Wang, L., Song, Y., \& Malow, B. A. (2012). A Pilot Study of Shoulder Placement for Actigraphy in Children. Behavioral Sleep Medicine, 1O(2), 138-147. https://doi.org/10.1080/15402002.2011.596598 Akbari Kamrani, A. A., Shams, A., Shamsipour Dehkordi, P., \& Mohajeri, R. (2014). The effect of low and moderate intensity aerobic exercises on sleep quality in men older adults. Pakistan Journal of Medical Sciences, 3o(2). https://doi.org/10.12669/ pjms.302.4386 actigraphy and sleep diaries is poor across all measures in aging adults with type 2 diabetes patients when they examined that on a sample of 53 T2D adults (aged 5076 years) were enrolled. Participants wore a wrist ActiGraph and filled out a daily sleep diary for eight days. Total sleep time (TST), sleep efficiency (SE), sleep onset latency (SOL), and wake after sleep onset (WASO) were obtained from the actigraphy and sleep diaries. Bland-Altman plots were conducted to examine the agreement between each sleep outcome.

\section{Limitations}

This study has several limitations. First, the sex differences in outcomes are representative of perioperative practice in Cairo. Similar research needs to be conducted in other settings to confirm the generalizability of our findings. Additionally, there is a huge gap in patient education and information about cardiac rehabilitation, many patients rejected to share in the study when they knew that they may do resistance exercises after $\mathrm{CABG}$, claiming that it is very risky exercise. Finally, such studies are by nature subject to residual confounding.

\section{Acknowledgment}

The authors acknowledge Assistant Prof. Dr Ashraf AbdElaal, Assistant Professor of physical therapy for cardiovascular/respiratory disorders and geriatrics, Cairo University, who assisted a lot in the statistical part of the results section.

\section{Funding Acknowledgment}

"The author acknowledges funding received from the European Society of Cardiology in form of an ESC Nursing Grant”.

\section{Conflict of interest}

The authors do not declare any conflict of interest.

Ancoli-israel, S., Cole, R., Alessi, C., Chambers, M., Moorcroft, W., \& Pollak, C. P. (2003). Cpr_Actigraphy, (April 2002).

Baraúna, V. G., Oliveira, E. M., Phillips, M. I., Negrão, C. E., \& Fernandes, T. (2015). Aerobic exercise training promotes physiological cardiac remodeling involving a set of microRNAs. American Journal of Physiology-Heart and Circulatory Physiology, 309(4), H543-H552. https://doi.org/10.1152/ajpheart.00899.2014

Barger, L. K., Rajaratnam, S. M. W., Cannon, C. P., Lukas, M. A., Im, 
K. A., Goodrich, E. L., ... O’Donoghue, M. L. (2017). Short sleep duration, obstructive sleep apnea, shiftwork, and the risk of adverse cardiovascular events in patients after an acute coronary syndrome. Journal of the American Heart Association, 6(10). https://doi.org/10.1161/JAHA.117.006959

Bohannon, R. W., \& Crouch, R. (2017). Minimal clinically important difference for change in 6-minute walk test distance of adults with pathology: a systematic review. Journal of Evaluation in Clinical Practice, 23(2), 377-381. https://doi.org/10.1111/ jep.12629

Bonardi, J. M. T., Lima, L. G., Campos, G. O., Bertani, R. F., Moriguti, J. C., Ferriolli, E., \& Lima, N. K. C. (2016). Effect of different types of exercise on sleep quality of elderly subjects. Sleep Medicine, 25, 122-129. https://doi.org/10.1016/j.sleep.2016.06.025

Chair, S. Y., Wang, Q., Cheng, H. Y., Lo, S. W. S., Li, X. M., Wong, E. M. L., \& Sit, J. W. H. (2017). Relationship between sleep quality and cardiovascular disease risk in Chinese post-menopausal women. BMC Women's Health, 17(1), 1-7. https://doi.org/10.1186/ s12905-017-0436-5

Chang, C.-H., Lin, K.-C., Ho, C.-S., \& Huang, C.-C. (2019). Accuracy of the energy expenditure during uphill exercise measured by the Waist-worn ActiGraph. Journal of Exercise Science \& Fitness, 17(2), 62-66. https://doi.org/10.1016/J.JESF.2019.01.003

Chen, L. J., Fox, K. R., Ku, P. W., \& Chang, Y. W. (2016). Effects of Aquatic Exercise on Sleep in Older Adults with Mild Sleep Impairment: a Randomized Controlled Trial. International Journal of Behavioral Medicine, 23(4), 501-506. https://doi. org/10.1007/s12529-015-9492-O

Chennaoui, M., Arnal, P. J., Sauvet, F., \& Léger, D. (2015). Sleep and exercise: A reciprocal issue? Sleep Medicine Reviews, 20, 59-72. https://doi.org/10.1016/J.SMRV.2014.06.008

Chien, M., \& Chen, H. (2015). Poor Sleep Quality is Independently Associated with Physical, 11(3).

de Vries, J. D., van Hooff, M. L. M., Geurts, S. A. E., \& Kompier, M. A. J. (2016). Exercise as an Intervention to Reduce Study-Related Fatigue among University Students: A Two-Arm Parallel Randomized Controlled Trial. PloS One, 11(3), e0152137. https://doi. org/10.1371/journal.pone.0152137

Fontes, F., Gonçalves, M., Maia, S., Pereira, S., Severo, M., \& Lunet, N. (2017). Reliability and validity of the Pittsburgh Sleep Quality Index in breast cancer patients. Supportive Care in Cancer, 25(10), 3059-3066. https://doi.org/10.1007/s00520-017-3713-9

Joseph, H. K., Whitcomb, J., \& Taylor, W. (2015). Effect of Anxiety on Individuals and Caregivers After Coronary Artery Bypass Grafting Surgery A Review of the Literature, (October), 10-13. https://doi.org/10.1097/DCC.oooooooooooo0137

Kline, C. E. (2014). The Bidirectional Relationship Between Exercise and Sleep: Implications for Exercise Adherence and Sleep Improvement. American Journal of Lifestyle Medicine, 8(6), 375-379. https://doi.org/10.1177/1559827614544437

Kovacevic, A., Mavros, Y., Heisz, J. J., \& Fiatarone Singh, M. A. (2018). The effect of resistance exercise on sleep: A systematic review of randomized controlled trials. Sleep Medicine Reviews, 39, 52-68. https://doi.org/10.1016/J.SMRV.2017.07.002

Landry, G. J., Best, J. R., \& Liu-Ambrose, T. (2015). Measuring sleep quality in older adults: A comparison using subjective and objective methods. Frontiers in Aging Neuroscience, 7(SEP), 1-10. https://doi.org/10.3389/fnagi.2015.00166

Lunsford-Avery, J. R., LeBourgeois, M. K., Gupta, T., \& Mittal, V. A.
(2015). Actigraphic-measured sleep disturbance predicts increased positive symptoms in adolescents at ultra high-risk for psychosis: A longitudinal study. Schizophrenia Research, 164(1-3), 15-20. https://doi.org/10.1016/J.SCHRES.2015.03.013

Mane, E., \& Memushaj, L. (2018). Luisiana Memushaj, (August), 83-90. https://doi.org/10.5281/zenodo.1412109

Manzar, M. D., BaHammam, A. S., Spence, D. W., Zannat, W., Moiz, J. A., Pandi-Perumal, S. R., \& Hussain, M. E. (2015). Validity of the Pittsburgh Sleep Quality Index in Indian University Students. Oman Medical Journal, 3o(3), 193-202. https://doi. org/10.5001/omj.2015.41

Mendelson, M., Borowik, A., Michallet, A., Perrin, C., \& Monneret, D. (2015). ORIGINAL RESEARCH, (6), 26-32. https://doi. org/10.1111/ijpo.12015

Mollayeva, T., Thurairajah, P., Burton, K., Mollayeva, S., Shapiro, C. M., \& Colantonio, A. (2016). The Pittsburgh sleep quality index as a screening tool for sleep dysfunction in clinical and non-clinical samples: A systematic review and meta-analysis. Sleep Medicine Reviews, 25, 52-73. https://doi.org/10.1016/J. SMRV.2015.01.009

Montgomery, P., Ja, D., Montgomery, P., \& Ja, D. (2009). Physical exercise for sleep problems in adults aged $60+$ ( Review ), (4). https://doi.org/10.1002/14651858.CDo03404.www.cochranelibrary.com

Nichols, C. (2015). Actigraphy : Validity, Reliability , and Clinical Utility.

Passos, G. S., Dalva, I. I., Rollemberg, L., Gonc, I. M., \& Tufik, I. I. S. (2012). Is exercise an alternative treatment for chronic insomnia?, 67(6), 653-659. https://doi.org/10.6061/clinics/2012(06)17

Peppard, P. E., Young, T., Barnet, J. H., Palta, M., Hagen, E. W., \& Hla, K. M. (2013). Increased prevalence of sleep-disordered breathing in adults. American Journal of Epidemiology, 177(9), 1006-1014. https://doi.org/10.1093/aje/kws342

Rn, Z. W., Rn, X. P., Rn, K. L., Rn, C. J. W., Intensive, M. N., \& Drhlthsc, C. (2019). Effects of combined aerobic and resistance training in patients with heart failure : A meta-analysis of randomized , controlled trials, (November 2018), 1-9. https://doi. org/10.1111/nhs.12593

Slightam, C., Keller, M., Kim, S., Bertram, F., Petrowski, K., Jamison, A. L., \& Roth, W. T. (2017). Assessing sleep quality using self-report and actigraphy in PTSD. Journal of Sleep Research, 27(3), e12632. https://doi.org/10.1111/jsr.12632

Stingeni, L., Raponi, F., Hansel, C., Agostinelli, D., \& Lisi, P. (2011). Le correlazioni clinico-anamnestiche e i risultati dei test cutanei allergodiagnostici nelle reazioni avverse cutaneo-mucose ad antibatterici sistemici. Annali Italiani Di Dermatologia Allergologica Clinica e Sperimentale, 65(1), 28-33. https://doi. org/10.1017/S174413741200015X

Tobaldini, E., Costantino, G., Solbiati, M., Cogliati, C., Kara, T., \& Nobili, L. (2017). Sleep, sleep deprivation, autonomic nervous system and cardiovascular diseases. Neuroscience \& Biobehavioral Reviews, 74, 321-329. https://doi.org/10.1016/J.NEUBIOREV.2016.07.004

Walsh, J., Eastwood, P. R., Straker, L. M., Slater, J. A., King, S., \& Botsis, T. (2015). Assessing sleep using hip and wrist actigraphy. Sleep and Biological Rhythms, 13(2), 172-180. https://doi. org/10.1111/sbr.12103

Yang, P., Ho, K., Chen, H., \& Chien, M. (2012). Exercise training im- 
proves sleep quality in middle-aged and older adults with sleep problems : a systematic review. Journal of Physiotherapy, 58(3), 157-163. https://doi.org/10.1016/S1836-9553(12)70106-6

Yang, P. L., Huang, G. S., Tsai, C. S., \& Lou, M. F. (2015). Sleep quality and emotional correlates in Taiwanese coronary artery bypass graft patients 1 week and 1 month after hospital discharge: A repeated descriptive correlational study. PLOS ONE, 1O(8), 1-16. https://doi.org/10.1371/journal.pone.0136431
Abbreviations:
1. CABG: coronary artery bypass grafting
2. AG: aerobic group
3. ARG: combined aerobic and resistance group
4. PSQI: Pittsburg sleep quality index
5. 6MWT: six minute walk test
6. INC: incorporated
7. USA: united states of America
8. SQ: sleep quality
9. CVD: cardiovascular disease
10. $\mathrm{N}$ : number
11. RPE: rate of perceived exertion
12. SD: standard deviation
13. UHR: ultra-high risk
14. TST : Total sleep time
15. SE : sleep efficiency
16. SOL : sleep onset latency
17. WASO : wake after sleep onset

Zhong, Q. Y., Gelaye, B., Sánchez, S. E., \& Williams, M. A. (2015). Psychometric Properties of the Pittsburgh Sleep Quality Index (PSQI) in a Cohort of Peruvian Pregnant Women. Journal of Clinical Sleep Medicine, 11(8), 869-877. https://doi.org/10.5664/ jesm.4936

Zhu, B., Bronas, U. G., \& Fritschi, C. (2018). Sleep assessment in aging adults with type 2 diabetes: agreement between actigraphy and sleep diaries. Sleep Medicine, 46, 88-94. https://doi.org/10.1016/J.SLEEP.2018.03.008 